\title{
COMPORTAMIENTO A LA FATIGA DE UN ACERO SAE 1022 RECUBIERTO CON CAPA CARBURADA OBTENIDO POR CEMENTACIÓN SÓLIDA
}

Responsable: Ing. Freddy Cori Nina Miembro: Ing. Julián Nieto Quispe

\section{RESUMEN}

Se ha investigado la influencia de la cementación sólida depositada en la superficie de un acero de bajo carbono SAE 1022, sobre el comportamiento al desgaste y a la fatiga en condiciones de flexión rotativa en ménsula. Los recubrimientos fueron obtenidos por el proceso de difusión e intercambio gas metal, utilizando como carburante carbón vegetal en un $80 \%$ y como activador el carbonato de bario en un $20 \%$. Los espesores de los recubrimientos o de la capa carburada obtenidos fueron: $0,554,0,738,2,343$ y $2,665 \mathrm{~mm}$. Mediante estudios fractográficos se observó que las grietas por fatiga tienen, generalmente, la zona de origen en la superficie del substrato o también en el recubrimiento. Sobre la base de un modelo experimental, se seleccionaron como parámetros de la cementación 900 ${ }^{\circ} \mathrm{C}$ y un tiempo de 8 horas. Luego un temple en agua seguido desde $800^{\circ} \mathrm{C}$ y $2 \mathrm{~h}$ de calentamiento previo. Finalmente, un revenido a $205^{\circ} \mathrm{C}$ durante $3 \mathrm{~h}$; con ello se obtuvo un incremento en el límite de fatiga muy significativo.

\section{ABSTRACT}

Has investigated the solid cementation's influence deposited on the surface of a low-carbon steel SAE 1022, on the behavior to the wear and to the fatigue. The coatings were obtained for the diffusion process gas metal, utilizing as fuel charcoal in one $80 \%$ and like activator $\mathrm{BaCO}_{3}$ in one $20 \%$. The thicknesses of the carbureted coatings obtained were : $0,554,0,738,2,343$ and $2,665 \mathrm{~mm}$. On the basis of an experimental model $900 \mathrm{oC}$ and 2 hours was selected as parameters of the cementation. Then the samples were heated 2 hours for $800 \mathrm{oC}$ and fast cooling in water. Finally the samples were tempered during 3 hours for $205 \mathrm{oC}$. These termic treatments increment significant- fatigue limit.

Palabras claves: Recubrimiento por cementación sólida, límite de fatiga, ensayos de tracción y fatiga, tratamientos superficiales.

\section{INTRODUCCIÓN}

El acero SAE 1022 es un acero de bajo carbono y es el que se ha utilizado en el presente trabajo. Previamente en la fábrica, el acero es laminado en calientea luego de ser enfriadas, las barras son decapadas en húmedo y sometidos a un trabajo en frío de estirado para ser calibradas a su dimensión y tolerancia final. Estas barras se caracterizan por su alta exactitud dimensional, buena calidad superficial El alto contenido de manganeso incrementa la dureza y la resistencia y puede ser tratado térmicamente, aunque poseen una relativa baja resistencia a la rotura por fatiga y al desgaste. Este hecho ha llevado al desarrollo de varios tipos de tratamientos superficiales con el fin de impartir mejores propiedades tribiológicas incrementando la resistencia a la fractura por fatiga, al desgaste y a la corrosión.

Entre los tratamientos que son es necesarios tener en cuenta por estar relacionados al presente trabajo de investigación, están los tratamientos mecánicos como el mecanizado, desbaste y pulido; los tratamientos térmicos tales como el endurecimiento por llama directa y por inducción, y los recubrimientos superficiales como la cementación en caja; la nitruración, el boronizado, etc. Es importante tener en cuenta que durante el tratamiento térmico en una atmósfera oxidante [20], los aceros pueden sufrir una descarburación de la superficie, reduciendo la resistencia a la fatiga por la pérdida del carbonosuperficial. Con el tratamiento de cementación en caja se aumenta la concentración de carbono superficial, mejorando la resistencia a la fatiga.

En relación con trabajos previos realizados en esta área, con aceros similares que utilizamos en el presente trabajo, Ludwik [20] investigó en un acero de bajo carbono con \% $\mathrm{C}=$ $0,17-0,19 \%$, y recocido a $900^{\circ} \mathrm{C}$, resultando que su estructura micrográfica estaba constituida por ferrita y perlita, carga de rotura $(R)=40-42,5$ $\mathrm{kg} / \mathrm{mm} 2$, dureza $\mathrm{HB}=112$ á 118 , y el límite de fatiga $(f)=23 \mathrm{~kg} / \mathrm{mm} 2$, donde comprueba que el límite de fatiga es óptimo.

Love [27] ha experimentado el efecto de la carburación en la resistencia a la fatiga de los aceros y en particular de un acero SAE 1015 con $0,16 \%$ de $\mathrm{C}$, sin y con tratamiento de carburación, concluyendo que para un acero $\operatorname{SAE} 1015(0,16 \% \mathrm{C})$, con un tratamiento de carburización es posible mejorar la resistencia a la fatiga. 
Es importante evaluar el efecto de los conjuntos substrato-recubrimiento sobre su comportamiento a la fatiga y al desgaste, que es nuestro tema de investigación y para el que hemos seleccionado el proceso de cementación sólida, utilizando como elemento difusor el carbono proveniente del carbón vegetal con adiciones de carbonato de bario como único activador $y$ sometidos a temperaturas entre 800 á $950{ }^{\circ} \mathrm{C}$ y como sustrato el acero SAE 1022 producido en Aceros Arequipa S. A. Se selecciona este proceso porque se utilizan materias primas existen en nuestro medio. La investigación nos permite conocer ¿cuál es la influencia de las variables del tiempo y temperatura en el proceso de cementación sobre la resistencia al desgaste y a la fatiga en el acero SAE 1022? Justificando así el presente trabajo, ya que contribuirá a mejorar y evaluar las propiedades mecánicas del acero en estudio Por tanto, la hipótesis del estudio es "La resistencia al desgaste y a la fatiga de un acero SAE 1022 aumentará en función del tiempo y temperatura de cementación". Para la evaluación de los resultados se utilizó el diseño factorial con dos factores y cuatro niveles de repetición; para el desarrollo del modelo se ha aplicado el software Statgraphics plus versión 4, del que resultó una ecuación que relaciona las variables temperatura, tiempo y espesor de la capa.

\section{MATERIALESYMÉTODOS}

Se mecanizaron especímenes, una cantidad de setenta y ocho unidades del substrato que es el acero SAE 1022 sin y con recubrimiento de cementación sólida. Las probetas se han constituido por diez y ocho especímenes de forma cilindrica de $40 \times 19 \mathrm{~mm}$, para los ensayos metalográficos, dureza, microdureza, estudio micrográfico y el espesor de los recubrimientos, doce probetas para los ensayos de tracción y cuarenta y ocho probetas para los ensayos de fatiga. La composición química nominal del acero SAE 1022 se presenta en la Tabla 2-1.

TABLA 2-1 Composición Química Nominal del Acero SAE 1022

\begin{tabular}{|l|l|l|l|l|}
\hline$\% C$ & $\% M_{n}$ & $\% p$ & $\% S$ & \%Fe \\
\hline $0,18-0,23$ & $0,70 \cdot 0,0,90$ & 0,04 máx. & $0,05 \%$ máx. & Balance \\
\hline
\end{tabular}

Fuente: Databook, editado por la American Society for Metals, 1988.

La composición química del acero utilizado como substrato se presenta en la Tabla 3-1.

TABLA 3-1 Composición química real del acero SAE 1022 utilizado en el proyecto

\begin{tabular}{|l|l|l|l|l|l|l|}
\hline$\% \mathrm{C}$ & $\% \mathrm{Mn}$ & $\% \mathrm{P}$ & $\% \mathrm{~S}$ & $\% \mathrm{Si}$ & $\%$ Impurezas & $\% \mathrm{Fe}$ \\
\hline 0,19 & 0,89 & 0,016 & 0,012 & 0,211 & 0,1695 & Balance \\
\hline
\end{tabular}

Fuente: Análisis químico Aceros Arequipa S. A.
Las propiedades mecánicas del acero según la norma SAE son, resistencia a la tracción $=49,0 \mathrm{~kg} / \mathrm{mm}^{2}$, límite de fluencia $=34,0 \mathrm{~kg} / \mathrm{mm}^{2}$ y el alargamiento en $50 \mathrm{~mm}=35 \%$.

Para el pulido se ha utilizado lijas de SiC desde $N^{\circ} 60$, hasta 1000 y pulido con paño y alúmina, que han permitido eliminar las muescas circunferenciales del mecanizado. Como cementante sólido se utilizó el carbón vegetal de $38006500 \mathrm{Kcal} / \mathrm{kg}$, exenta de humedad, la granulometria utilizada fue de $39 \mathrm{~mm}$ de diámetro. Como único activador utilizado ha sido el carbonato de bario comercial. Se procedió a los ensayos: metalográficos en el microscopio metalográfico NEOPOT 21, Reichert Metavar de 1000 aumentos y el microscopio marca NIKON digital, para los ensayos de microdureza se utilizó un microdurómetro marca CHIMADZU en microdureza Vickers.; el ensayo de tracción se utilizó en un equipo marca KRATOS de 20 TM con un equipo de cómputo $\mathrm{y}$, finalmente, el ensayo de fatiga se realizó en un equipo de ensayo de fatiga por flexión rotativa en ménsula, para el tratamiento de carburización se utilizó el horno eléctrico de mufla marca Heraeus electrónic, con un rango de temperatura de 0 a $1000^{\circ} \mathrm{C}$.

La cementación se realizó de acuerdo con el modelo experimental trazado, como se indica en la tabla $2-5$, considerando las variables de temperatura de cementación y el tiempo de permanencia, es decir, el diseño factorial propuesto a dos niveles $2 \mathrm{k}$ distribuido de la manera siguiente:

Terminado este ciclo de pruebas, con los datos obtenidos, nos permite analizar estos datos y tomar las variables de $900^{\circ} \mathrm{C}$ y $8 \mathrm{~h}$ con tres repeticiones más y asi obtener la capa de recubrimiento óptima.

\section{RESULTADOS YDISCUSIÓN}

\subsection{Características de los Recubrimientos}

\subsubsection{Determinación de las condiciones óptimas de Tiempo y Temperatura de Carburización, Según el Modelo Superficie Respuesta.}

A partir de la tabla 2-5, hemos utilizado un modelo estadistico experimental, que relaciona el tiempo(t), la temperatura(T) y la capa $X$ de carburización y constituye una buena alternativa para resolver este problema de optimización. A través del microscopio metalográfico Metavar y Neophot 21 y obtuvimos las resultados que nos permitieron obtener la Tabla 4-2. La capa cementada se calculó a partir de las 
mediciones iniciales en milimetros de cada probeta de la tabla 4-2; con estos resultados se determinó el modelo matemático para el cálculo de la capa cementada que se expresa en la relación siguiente:

\section{Capa cementada $=2,76269-0,00259618^{*}$}

Temperatura - 2,07495

*Tiempo +0,00238929*temperatura*Tiempo.

Resumiendo se tiene:

$X=2,76269-0,00259618 .(T)-2,07495 .(t)+0,00238929 .(T) .(t) 4-2$ Donde:

$X=$ Espesor de la capa carburada en $\mathrm{mm}$.

$\mathrm{t}=$ tiempo de carburización en horas.

$\mathrm{T}=$ Temperatura de carburización en ${ }^{\circ} \mathrm{C}$

\begin{tabular}{|l|l|l|r|r|r|}
\hline \multicolumn{3}{|c|}{$\begin{array}{c}\text { Tabla 2 - } 5 \text { Matriz de diseño con } \\
\text { valores reales para pruebas }\end{array}$} & \multicolumn{3}{c|}{$\begin{array}{c}\text { Tabla 4 - 2 Resultados del } \\
\text { diseño experimental global. }\end{array}$} \\
\hline $\mathbf{N}^{\circ}$ Prueba & $\begin{array}{c}\text { Tiempo } \\
(\mathrm{h})\end{array}$ & $\begin{array}{c}\text { Temperatura } \\
\left({ }^{\circ} \mathrm{C}\right)\end{array}$ & $\begin{array}{c}\text { Temperatura } \\
\left({ }^{\circ} \mathrm{C}\right)\end{array}$ & Horas & $\begin{array}{c}\text { Capa } \\
\text { calculada }\end{array}$ \\
\hline 1 & $4(-)$ & $880(-)$ & 950 & 4 & 1,108482 \\
\hline 2 & $12(+)$ & $880(-)$ & 880 & 12 & 0,8398084 \\
\hline 3 & $4(-)$ & $950(+)$ & 880 & 4 & 0,6188068 \\
\hline 4 & $12(+)$ & $950(+)$ & 950 & 12 & 2,667486 \\
\hline $\mathbf{N}^{\circ}$ de \\
probetas & $\begin{array}{l}\text { Tiempo } \\
(\text { Horas) }\end{array}$ & $\begin{array}{c}\text { Temperatura } \\
\left({ }^{\circ} \mathrm{C}\right)\end{array}$ & 900 & 8 & 1,060358 \\
\hline 5 & 8 & 900 & 900 & 8 & 1,060358 \\
\hline 6 & 8 & 900 & 900 & 8 & 1,060358 \\
\hline 7 & 8 & 900 & 925 & 4 & 0,933598 \\
\hline
\end{tabular}

En el Gráfico 4.1 se muestra una representación del gráfico de la superficie respuesta mediante la curva de isovalores del espesor de la capa versus las variables tiempo y la temperatura, donde se pueden observar las regiones para determinar el grosor de la capa cementada para este acero. Esta representación ayuda a buscar el tratamiento óptimo en cuanto a espesor en función del tiempo y de la temperatura, sin olvidar que no se conoce la calidad y la tenacidad de la capa formada en las condiciones que se hayan elegido. Para un tratamiento de carburización a $900{ }^{\circ} \mathrm{C}$ y $8 \mathrm{~h}$ se tiene la capa conveniente con un espesor de 0,8 1,0 la franja de color amarillo y que tiene una tendencia a bajar el tiempo de tratamiento.

GRÁFICO 4.1 Curva de Isovalores del Espesor de Capa en Función del Tiempo y temperatura de Tratamiento.

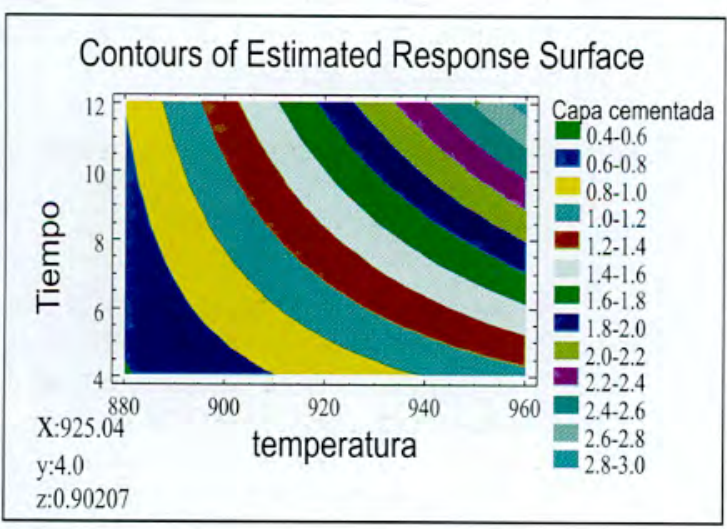

Donde podemos concluir que en este método la temperatura tiene mayor influencia en la formación del espesor de la capa carburada que el tiempo.

\subsubsection{Efecto de la Dureza}

La determinación de la profundidad de penetración de la dureza en el acero se realizó con apoyo del microdurómetro marca Chimadzu tipo $\mathrm{M}$, modelo $\mathrm{N}^{\circ} 79307$ con cargas de 100 a 400 gramos. La curva de penetración de la dureza puede determinarse mediante medidas de la microdureza a diferentes distancias crecientes de la superficie como se presenta en el Gráfico 4.4 que es la curva de dureza Vickers a una probeta con tratamiento a $880^{\circ} \mathrm{C}$ y el tiempo de 4 horas. Se han tomado lecturas de durezas de todas las probetas Para obtener las microdurezas HV se ha utilizado la ecuación siguiente:

$$
H V=1854,4 \begin{aligned}
& P \\
& d^{2}
\end{aligned}
$$

Donde $\mathrm{P}=$ carga $300 \mathrm{gr}$ en forma constante; $\mathrm{d}^{2}=$ en micras. Yel Gráfico 4.4 muestra la curva: distancia a la superficie $(\mathrm{mm})$ Dureza Vickers $\left(\mathrm{Kp} / \mathrm{mm}^{2}\right)$, trazada a partir de la Tabla 4-4 . Aquí se observa que la dureza en el borde para esta probeta es de $319.9 \mathrm{HV}$ y en el núcleo o cercano al núcleo o profundidad ideal es $145.2 \mathrm{HV}$; para la dureza eficaz se toma el valor medio de las microdurezas y corresponde a 179,3 HV la mismia que está a una distancia aproximada desde el borde hacia el núcleo de $0,85 \mathrm{~mm}$ según la norma DIN 17014.

Se ha resumido en una tabla donde se presentaron las microdurezas tomadas a las nueve probetas carburadas bajo distintas condiciones y según el modelo planteado en la Tabla 2-5.. La probeta 7 tiene un tratamiento de carburización a $900{ }^{\circ} \mathrm{C}$ y durante $8 \mathrm{~h}$. se puede observar que las durezas son en el borde de 315,4 HV, la dureza eficaz es 195,8 HV y la dureza en el núcleo de $154,4 \mathrm{HV}$, y para este tipo de tratamiento el espesor de la capa cementada es de $1,250 \mathrm{~mm}$.

\subsubsection{Efecto del Tratamiento de carburización Sobre la Microestructura del Sustrato Base}

Los aceros de bajo carbono como los SAE 1022, que es motivo de estudio, en estado de recepción presentan una estructura de matriz ferrítica c.c. y un grado de solubilidad del carbono en el $\mathrm{Fe}$ alfa, ya que contiene $0,19 \%$ de carbono. La microfotografía tomada en el laboratorio a probetas de corte longitudinal a $100 \mathrm{x}$ aumentos, de la barra de $19 \mathrm{~mm}$ del acero SAE 1022, Ataque: 
Nital $3 \%$, presenta una estructura constituida por ferrita proeutectoide en aproximadamente un $75 \%$ que son áreas de coloración blanca y de perlita de aproximadamente un $25 \%$ indicada en las áreas oscuras. La estructura es heterogénea, de granos ligeramente alargados en la dirección longitudinal, lo que es característico de las aleaciones con adiciones de manganeso; se estima aproximadamente el tamaño de grano medio o normal de 6 ASTM. En la industria automotriz se utilizan los granos con 4, 5, 6, y 7 ASTM, de preferencia los de 5 a 7 ASTM. Además se observa ferrita Widmanstatten.

TABLA 4-4 Microdurezas de la probeta carburada a $880 \mathrm{C}$ y $4 \mathrm{~h}$

\begin{tabular}{|c|c|c|c|}
\hline $\begin{array}{c}\text { LECTURA } \\
\mathbf{N}^{0}\end{array}$ & $\begin{array}{c}\text { DISTANCIA } \\
(\mathbf{m m})\end{array}$ & $\begin{array}{c}\text { DIAGONAL } \\
\text { (Micras) }\end{array}$ & $\begin{array}{c}\text { DUREZ } \\
\mathbf{A}(\mathbf{H V})\end{array}$ \\
\hline $\mathbf{1}$ & 0,10 & 41,7 & 319,9 \\
\hline $\mathbf{2}$ & 0,25 & 4,5 & 323,0 \\
\hline $\mathbf{3}$ & 0,40 & 43,3 & 29,7 \\
\hline $\mathbf{4}$ & 0,55 & 45,3 & 271,1 \\
\hline $\mathbf{5}$ & 0,70 & 49,6 & 226,1 \\
\hline $\mathbf{6}$ & 0,85 & 55,7 & 179,3 \\
\hline $\mathbf{7}$ & 1,00 & 58,8 & 160,9 \\
\hline $\mathbf{8}$ & 1,15 & 58,5 & 162,6 \\
\hline $\mathbf{9}$ & 1,30 & 60,3 & 153,0 \\
\hline 10 & 1,45 & 61,8 & 145,7 \\
\hline $\mathbf{1 1}$ & 1,60 & 61,9 & 145,2 \\
\hline
\end{tabular}

Gráfico 4.4 Gráfica de microdurezas con valores de la Tabla 4-4
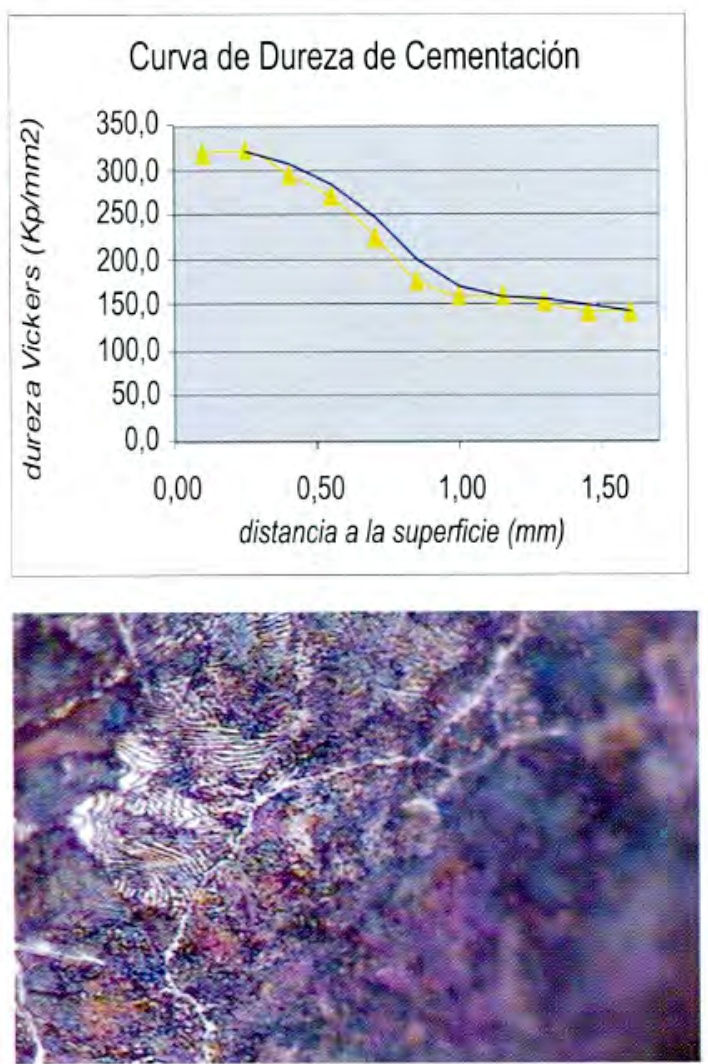

Microestructura del borde a 400x aumentos (Muestra 3)

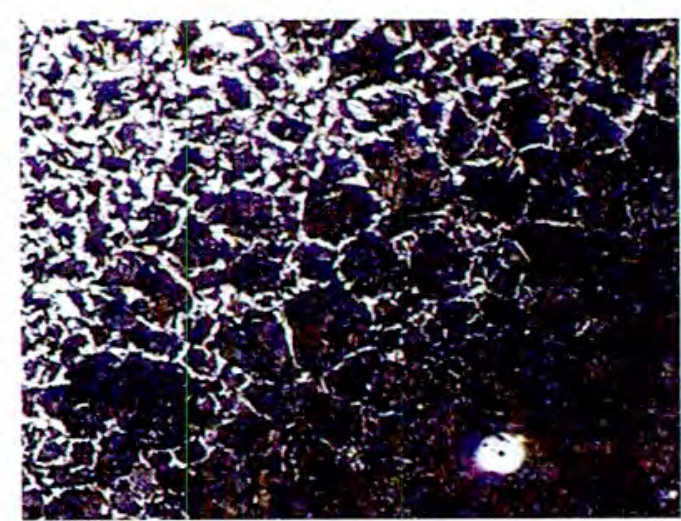

Microestructura del medio a 100x ( Muestra 3)

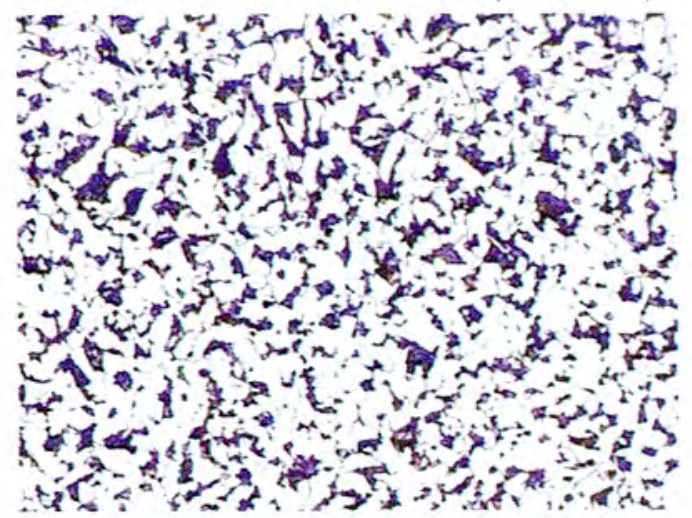

Microestructura del centro a 100x (Muestra 3)

Fotos 4.10. Probeta 5: Carburizado a $900^{\circ} \mathrm{C}$ y 8 horas.

Podemos concluir que el efecto que tiene la cementación sobre la microestructura del sustrato base es que se distinguen 3 zonas: Ejemplo en las micrografias de la Foto 4.10, se observan tres zonas bien diferenciadas: la del borde o zona hipereutectoide que tiene una microestructura de perlita con una red blanca de cementita y con grano grueso; la siguiente zona eutectoide, en el centro hacia el núcleo de mucho menor en tamaño de grano, compuesta de perlita y, finalmente, la zona hipoeutectoide de perlita y ferrita, con la cantidad de ferrita aumentando hasta que se alcanza la parte interna que es el núcleo. La formación de la microestructura está en función de la temperatura y el tiempo; una alta temperatura producirá un tamaño de grano grande y la probeta será frágil; el tiempo tiene una significación menor; por consiguiente, es conveniente que la temperatura y el tiempo sean los óptimos para producir la microestructura, dureza y tenacidad requeridas.

\subsection{Resultados de los Ensayos Mecánicos}

\subsubsection{Ensayo de Tracción}

Los ensayos de tracción se realizaron en una máquina de ensayo marca KRATOS de 20 TM y el tipo de probeta utilizada fue el de la Norma Americana B557M-ASTM. 
3.2.1.1. Ensayo de Tracción sin Tratamiento de Carburización

El ensayo de tracción sin recubrimiento se realizó luego de mecanizar el material a sus dimensiones estándar, luego se procedió al ensayo en equipos de una capacidad de 20TM; al finalizar el ensayo de todas las probetas se han obtenido valores que este acero tiene gran ductilidad puesto que la fractura de la probeta ha sido en copa y cono; la estricción ha tenido un valor de $59 \%$, la elongación de un $21,4 \%$ en $50 \mathrm{~mm}$, la resistencia máxima de un $64,55 \mathrm{Kg} / \mathrm{mm}^{2}$ y la tensión de rotura en un $38,2 \mathrm{Kg} / \mathrm{mm}^{2}$.

\subsubsection{Ensayo de Tracción a Probetas Cementadas}

El ensayo se realizó a probetas con tratamiento de carburización a $925^{\circ} \mathrm{C}$ y 4 horas, luego un calentamiento a $800^{\circ} \mathrm{C}$ durante 2 horas y temple en agua y, finalmente, un revenido a $205^{\circ} \mathrm{C}$ durante 3 horas. Los resultados son que tiene gran dureza, fragilidad y tenacidad, la estricción ha tenido un valor de $0,69 \%$, la elongación de un $0,2 \%$ en $50 \mathrm{~mm}$, la resistencia máxima de un $66,09 \mathrm{~kg} / \mathrm{mm}^{2}$. $\mathrm{Se}$ puede concluir que debido al tratamiento de carburación aumenta la dureza, la estricción, elongación muy ligeramente; la resistencia máxima es también mayor, lo que le da una mayor tenacidad y cierto grado de fragilidad.

\subsubsection{Resultados de los Ensayos de Fatiga}

Los ensayos de fatiga se ejecutaron en una máquina de ensayo de fatiga por flexión rotativa en ménsula, siendo la probeta de la norma AIR 0830, normalizada (Francia) que resulta idóneo para simular dichas cargas alternativas en servicio.

3.2.2.1. Ensayo de Fatiga por Flexión Rotativa a Aceros Sae 1022 sin recubrimiento.

Este ensayo se realizó en un equipo de fatiga tipo flexión rotativa en ménsula, al finalizar nos da los valores indicados en la tabla 4-11 en $\mathrm{kg}(\mathrm{S})$ Ciclos(N) y con estos datos se transformó la fuerza $(\mathrm{kg})$ a esfuerzo de $\mathrm{kg} / \mathrm{mm}^{2}$ el gráfico 4-14 (inferior), donde se nota que la carga mínima es de $25 \mathrm{~kg}(\mathrm{~S}) 1028$ 230(N) ciclos; este punto representa el límite de fatiga debido a que la probeta no se fracturó en ese punto. En el Gráfico 4-12 se aprecia que el límite de fatiga para el acero SAE 1022 sin recubrimiento es de $70,1 \mathrm{~kg} / \mathrm{mm}^{2}$ y los ciclos son 1028 230.

La carga aplicada para el conjunto de ensayos fue de un máximo de $45 \mathrm{~kg}$ y esta fué descendiendo hasta $25 \mathrm{~kg}$, ya que en ese punto no se fracturó, por consiguiente, es el límite de resistencia a la fatiga para el acero SAE 1022 sin tratamiento.

\subsubsection{Ensayo de fatiga por flexión rotativa en ménsula del acero SAE 1022 con tratamiento de carburación.}

Las probetas son cementadas a $900^{\circ} \mathrm{C}$ y $8 \mathrm{~h}$ de tratamiento, posteriormente se les sometió a un temple en agua con un calentamiento a $800^{\circ} \mathrm{C}$ y una duración de $2 \mathrm{~h}$ y, finalmente, un revenido a $205^{\circ} \mathrm{C}$ durante $3 \mathrm{~h}$.

Luego de finalizar el ensayo de fatiga con todas las probetas se muestran todos los resultados en la Tabla 4-12, en $\mathrm{kg}(\mathrm{S})$ y ciclos(N) y en el Gráfico 4.14(superior), también para este gráfico se transformó a $\mathrm{kg} / \mathrm{mm} 2$, donde se puede observar que el limite de fatiga aumenta a $32 \mathrm{~kg}(\mathrm{~S})$ y $1256857(\mathrm{~N})$ ciclos o equivalente al esfuerzo $90.01 \mathrm{~kg} / \mathrm{mm} 2$ y $1256857(\mathrm{~N})$ ciclos, aumentando el límite de fatiga. Con una carga de $32 \mathrm{~kg}$ no se rompió, lo que nos da el límite de resistencia a la fatiga para el mismo acero SAE 1022, pero en particular y con capa cementada a $900^{\circ} \mathrm{C}$ y $8 \mathrm{~h}$ de tratamiento. En el diagrama se aprecia que el límite de duración o de fatiga corresponde a la carga de $32 \mathrm{~kg}$.

\begin{tabular}{|c|c|c|c|c|c|c|c|}
\hline \multicolumn{4}{|c|}{$\begin{array}{l}\text { Tabla } 4 \text {-11 Ensayo de fatiga a probetas de } \\
\text { acero SAE } 1022 \text { sin recubrimiento. }\end{array}$} & \multicolumn{4}{|c|}{$\begin{array}{l}\text { Tabla } 4 \text { - } 11 \text { Ensayo de fatiga a probetas de } \\
\text { acero SAE } 1022 \text { sin recubrimiento. }\end{array}$} \\
\hline $\begin{array}{c}N^{*} \\
\text { Probeta }\end{array}$ & $\begin{array}{l}\text { Carga } \\
\mathbf{S}(\mathbf{K g})\end{array}$ & $\begin{array}{l}\text { Ciclos } \\
N\end{array}$ & Fractura & $\begin{array}{c}N^{\infty} \\
\text { Probeta }\end{array}$ & $\begin{array}{l}\text { Carga } \\
\mathbf{S}(\mathrm{Kg})\end{array}$ & $\begin{array}{c}\text { Ciclos } \\
N\end{array}$ & Fractura \\
\hline 1 & 35 & 8682 & $\mathrm{Si}$ & 1 & 45 & 19337 & $\mathrm{Si}$ \\
\hline 2 & 35 & 5216 & $\mathrm{Si}$ & 2 & 45 & 30225 & Si \\
\hline 3 & 35 & 5062 & Si & 3 & 45 & 32187 & Si \\
\hline 4 & 30 & 22710 & $\mathrm{Si}$ & 4 & 44 & 35011 & Si \\
\hline 5 & 30 & 25526 & Si & 5 & 43 & 36225 & Si \\
\hline 6 & 30 & 31760 & Si & 6 & 40 & 36992 & Si \\
\hline 7 & 40 & 2160 & Si & 7 & 40 & 47823 & Si \\
\hline 8 & 40 & 1563 & $\mathrm{Si}$ & 8 & 40 & 63909 & $\mathrm{~s}$ \\
\hline 9 & 40 & 1156 & $\mathrm{Si}$ & 9 & 35 & 141728 & Si \\
\hline 10 & 45 & 1685 & $\mathrm{Si}$ & 10 & 35 & 145858 & Si \\
\hline 11 & 43 & 1296 & Si & 11 & 35 & 232793 & Si \\
\hline 12 & 44 & 1452 & si & 12 & 33 & 1162894 & si \\
\hline 13 & 25 & 1028230 & No & 13 & 32 & 1256857 & No \\
\hline 14 & 27,5 & 435200 & Si & \multirow{3}{*}{\multicolumn{4}{|c|}{$\begin{array}{l}\text { El valor del esfuerzo se ha calculado por la } \\
\text { ecuación } \quad f=\frac{M r}{t}\end{array}$}} \\
\hline 15 & 27,5 & 253075 & Si & & & & \\
\hline 16 & 27,5 & 288430 & $\mathrm{Si}$ & & & & \\
\hline
\end{tabular}

Gráfico 4.14 se expresa la en tensión $\mathrm{kg} / \mathrm{mm}^{2}$ (S) y ciclos $(\mathrm{N})$ se puede distinguir en este gráfico lo siguiente:

En tensión $70,1 \mathrm{~kg} / \mathrm{mm}^{2}$ (S) y 1028230 ciclos $(\mathrm{N})$ y en carga $25 \mathrm{~kg} \sin$ tratamiento.

En tensión $90,01 \mathrm{~kg} / \mathrm{mm}^{2}$ y $1256857(\mathrm{~N})$; en carga $32 \mathrm{~kg}$ con tratamiento. En ambos casos el incremento en el límite de resistencia a la fatiga es de un $28 \%$ aproximadamente. 
GRÁFICA 4.14 Curvas de Wholer Comparativas entre El Acero Sae 1022 con Tratamiento y sin Tratamiento de Cementación.

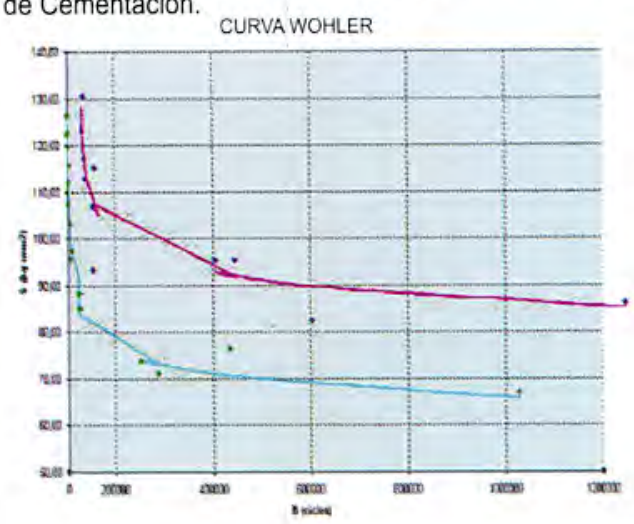

Estos resultados permiten concluir que el acero SAE 1022 sin tratamiento de cementación tiene un el limite de fatiga de 25 $\mathrm{kg}(\mathrm{S}) 1028230$ (N) ciclos ó 70,1kg/mm y 1 028230 ciclos; las mismas probetas, pero con un tratamiento de cementación a $900^{\circ} \mathrm{C}$ y un tiempo de $8 \mathrm{~h}$, templada y revenida, el limite de fractura a la fatiga aumentó ligeramente a 32 $\mathrm{kg}(\mathrm{S})$ y $1256857(\mathrm{~N})$ ciclos, es decir, 90,01 $\mathrm{kg} / \mathrm{mm}^{2}$ y $1256857(\mathrm{~N})$ ciclos. Por consiguiente, en las condiciones ya explicadas anteriormente y en condiciones severas de solicitaciones dinámicas el acero SAE 1022 con tratamiento será capaz de resistir una vida en servicio estimada por encima de los 10 años sin presentar ningún tipo de fallo; a diferencia de este mismo acero pero sin tratamiento. Lo que se concluye, comprobando la afirmación de la hipótesis del presente trabajo, es que la resistencia al desgaste y a la fatiga de un acero SAE 1022 aumentará en función del tiempo y temperatura de cementación.

\section{CONCLUSIONES}

1. Se ha investigado la influencia del recubrimiento por cementación sólida depositada en la superficie del acero SAE 1022, sobre el comportamiento al desgaste y a la fatiga por flexión rotativa en ménsula.

2. Las variables influyentes de la cementación son la temperatura y el tiempo de cementación, los cuales influirán posteriormente en la dureza, el tamaño de grano y el espesor de la capa cementada óptima.

3. Experimentalmente por el estudio realizadose muestra que la cementación sólida depositada en la superficie del acero SAE 1022, producido en el Perú, aumenta la resistencia al desgaste y la resistencia a la fractura por fatiga, relacionando la capa de cementación y las variables de temperatura y el tiempo.

4. Con la capa carburada en la superficie del acero, la resistencia a la fatiga mejoró significativamente.las condiciones operacionales fueron: cementación:a temperatura de $900^{\circ} \mathrm{C}$ y tiempo de 8 horas; temple en agua: a $800^{\circ} \mathrm{C}$; calentamiento de 2 horas; revenido: a $205{ }^{\circ} \mathrm{C}$ durante 3 horas; carburante: carbón vegetal $80 \%$ y carbonato de bario $20 \%$ y granulometría del carbón de 3 a $9 \mathrm{~mm}$. La dureza varia del borde al núcleo de mayor a menor, con un tamaño de grano medio.

5. La cementación sólida, es un proceso de difusión, simple y económico, ya que todos los insumos existen en nuestro medio.

6. El acero propuesto, con tratamiento, puede soportar condiciones severas de trabajo dinámico superando el tiempo de vida proyectado sin riesgo de falla. Su límite de fatiga supera considerablemente con respecto al mismo acero sin tratamiento.

7. La aplicación de la carburización en el ámbito industrial contribuirá a mejorar la calidad del acero en mecanización de piezas en el sector metal mecánico y además en parte sustituirá a la importación de este acero, con el consiguiente ahorro de divisas.

\section{v. BIBLIOGRAFÍA}

Schey Jhon A., Procesos de manufactura McGraw-Hill Ediciones, $3^{\circ}$ edición,México, 2002 (100-103).

A. M. Afanásiev V. A. Marien, Prácticas de laboratorio resistencia de materiales. Editorial MIR, Moscú, 1978; 42,43.

D. Grimberg (1989), Tratamiento térmico de aceros y sus prácticas de laboratorio. Editorial Limusa, $165-171 \mathrm{pp}$.

R. J. Love, The influence of surface condition on the fatigue strength of steel. Ohio, 1950;211-214.

Callister, JR. W. (1995), Introducción a la ciencia e ingenieria de los materiales. Editorial Reverte S.

A, España, 96-97 pp.

Apraiz Barreiro J., Tratamiento térmico de los aceros. Editorial DOSSAT, $9^{\circ}$ Edición, Madrid, 1990; 546-551.

Cazaud R., La fatiga de los metales. Editorial Aguilar, Madrid, 1977 (187-1194). 